\title{
Social activity method (SAM): A fractal language for mathematics
}

\author{
Paul Dowling
}

Received: 24 July 2012 / Revised: 17 November 2012 / Accepted: 12 February 2013 /

Published online: 13 March 2013

(C) The Author(s) 2013. This article is published with open access at Springerlink.com

\begin{abstract}
In this paper I shall present and develop my organisational language, social activity method (SAM), and illustrate some of its applications. I shall introduce a new scheme for modes of recontextualisation that enables the analysis of the ways in which one activity - which might be school mathematics or social research or any empirically observed regularity of practice - recontextualises the practice of another and I shall also present, deploy, and develop an existing scheme - domains of action in an analysis of school mathematics examination papers and in the structuring of what I refer to as the esoteric domain. This domain is here conceived as a hybrid domain of, first, linguistic and extralinguistic resources that are unambiguously mathematical in terms of both expression and content and, second, pedagogic theory often tacit - that enables the mathematical gaze onto other practices and so recontextualises them. A second and more general theme that runs through the paper is the claim that there is nothing that is beyond semiosis, that there is nothing to which we have direct access, unmediated by interpretation. This state of affairs has implications for mathematics education. Specifically, insofar as an individual's mathematical semiotic system is under continuous development - the curriculum never being graspable all at once - understanding - as a stable semiotic moment - of any aspect or object of mathematics is always localised to the individual and is at best transient, and the sequencing of such moments may well also be more individualised than consistent in some correspondence with the sequencing of the curriculum. This being the case, a concentration on understanding as a goal may well serve to inhibit the pragmatic acquisition and deployment of mathematical technologies, which should be the principal aim of mathematics teaching and learning. The paper is primarily concerned with mathematics education. SAM, however, is a language that is available for recruiting and deploying in potentially any context as I have attempted to illustrate with some of the secondary illustrations in the text.
\end{abstract}

P. Dowling $(\square)$

Institute of Education, University of London, London, UK

e-mail: P.Dowling@ioe.ac.uk 
Keywords Sociology·Social activity method-Discursive saturation - Esoteric domain · Recontextualisation

\section{Forensics and the myth of reference}

There is a marked tendency in social and educational research to promote what I have called the myth of reference (Dowling 1998), which is to say, to present itself as if it were about something other than itself, as if the language of the research represents objects and activities that the research has uncovered, but that, in principle, are accessible to all of us. Having represented the world, the mechanisms articulating the representation are pushed into the represented world and represented as causal structures. I call this forensics (Dowling 2009). The research game proceeds as a wrestle over which language constitutes the best representation, which structures the most deserving causes. I want to take a different tack. This paper presents an organisational language - SAM - that is about itself. This is achieved within a general approach that I refer to as constructive description (Dowling 1998, 2009) on which a little more later. Having announced this, I need to address two urgent issues. First, I do not mean to claim (or even aspire to) solipsism. I must establish an exteriority, which I shall refer to as the empirical setting (Dowling and Brown 2010). I will name the setting "knowledge," for the time being. This minimal detail is sufficient to give me a bearing on what lies beyond my constructions. The outcome of my engagement with the setting, however, is not a representation, but a recontextualisation so that, ultimately, "knowledge" becomes incorporated into my language.

Second, I must explain the significance of the term "for" in my title, because it may seem to signal a dualism that I appear to be denying. There is, however, no denial. Mathematics may be said, here, to stand for my audience; then "for" constitutes my language as a gift, the value of which is to be determined by that audience in pragmatic terms (and here Rorty (1989) and Schön (1987) and perhaps Carr and Kemmis (1986) are inspirational): Is it useful? If it is useful, then it might be fetched into mathematical practice, which move will entail another recontextualisation. Naturally, I shall attempt to market the language by offering some illustrations of SAM's use.

A second and associated tendency in educational research is the totalising of the represented in conformity with the representation. Here is Basil Bernstein on mathematics and other knowledge structures:

It might be useful here to make a distinction within horizontal knowledge structures, distinguishing those whose languages have an explicit conceptual syntax capable of 'relatively' precise empirical descriptions and/or of generating formal modeling of empirical relations, from those languages where these powers are much weaker. The former I will call strong grammars and the latter weak grammars. It is important to add here that 'strong' and 'weak' must be understood as relative within horizontal knowledge structures. From this point of view, economics, linguistics and parts of psychology would be examples of strong grammar. Mathematics would also be considered a horizontal knowledge structure 
as it consists of a set of discrete languages, for particular problems. Thus, mathematics and logic would be regarded as possessing the strongest grammers [sic], although these languages, for the most part, do not have empirical referents nor are they designed to satisfy empirical criteria. Examples of weak grammars would be sociology, social anthropology, and cultural studies.

(Bernstein 1999; 164)

The two characteristics of mathematics are, first, that it is a horizontal knowledge structure, that is, it consists of "a series of specialised languages with specialised modes of interrogation and criteria for the construction and circulation of texts" (Bernstein 1999; 162). This contrasts with a hierarchical knowledge structure that "takes the form of a coherent, explicit, systematically principled structure, hierarchically organized" (Bernstein 1996; 171). Second, mathematics has a strong grammar, although it is not entirely clear what the latter means given that it is defined above in relation to the precision of empirical descriptions, whilst mathematics itself is denied empirical referents. I shall return to this later. Meanwhile, here is Raymond Duval distinguishing between mathematics and "other domains of scientific knowledge," totalising both:

From an epistemological point of view there is a basic difference between mathematics and the other domains of scientific knowledge. Mathematical objects, in contrast to phenomena of astronomy, physics, chemistry, biology, etc., are never accessible by perception or by instruments (microscopes, telescopes, measurement apparatus). The only way to have access to them and deal with them is using signs and semiotic representations. That means that we have here only a single access to the knowledge objects and not a double access, mainly non-semiotic and secondarily semiotic, as is the case in the other areas.

(Duval 2006; 107)

Further:

Mathematics is the domain within which we find the largest range of semiotic representation systems, both those common to any kind of thinking such as natural language and those specific to mathematics such as algebraic and formal notations. And that emphasizes the crucial problem of mathematics comprehension for learners. If for any mathematical object we can use quite different kinds of semiotic representation, how can learners recognize the same represented object through semiotic representations that are produced within different representation systems?

(Duval 2006; 108)

The second of these extracts illustrates Duval's (2006) forensic action. First, mathematics is represented as a domain that mediates its objects through multiple semiotic systems. This is a structural feature of Duval's representation that is pushed into the empirical as a structural feature of mathematical knowledge. Second, this being the case, aspiring acquirers of mathematical knowledge are potentially disabled by this multiplicity. Now, adopting my pragmatic principle of evaluation, the forensic nature of the argument may not matter to the extent that the result proves to be useful 
in mathematics education. Forensic action - the pushing of a representation into a supposedly represented setting - may, however, distract us from the representation itself. To explain what I mean, I want to return to the first of the Duval extracts in which, in effect, he distinguishes between two modes of knowledge, one of which characterising "other domains of scientific knowledge" - provides access to its objects either by direct perception or via intermediary technology that does not function semiotically, that is, is semiotically inert. The other form of knowledge mathematics - mediates its objects via (multiple) semiotic representations. I shall explore the first case through an example from astrophysics as another domain of scientific knowledge. Here is the abstract of my exemplar paper to provide a sense of the nature of astrophysical representation (please bear with me!).

Photometric redshifts can be routinely obtained to accuracies of better than 0.1 in $\Delta z /(1+z)$. The issue of dust extinction, however, is one that has still not been well quantified. In this paper the success of two template-fitting photometric redshift codes (IMPZ and HYPERZ) at reliably returning $A_{V}$ in addition to redshift is explored. New data on the 2nd Canadian Network for Observational Cosmology (CNOC2) spectroscopic sample of $0.2<\mathrm{z}<0.7$ galaxies are presented. These data allow us to estimate $A_{V}$ values from the observed Balmer decrements. We also investigate whether the empirical value of $\gamma=0.44$, the ratio between gas- and star-derived extinction, as determined by Calzetti, is necessarily the best value for this sample.

When comparing the two codes with the Balmer-derived $A_{V}\left(\right.$ Balmer $\left.\left[A_{V}\right]\right)$, a correlation between the photometrically derived $A_{V}\left(\operatorname{Phot}\left[A_{V}\right]\right.$ ) and the Balmer $\left[A_{V}\right]$ is found. The correlation is improved when the empirical value of $\gamma$ of 0.44 is allowed to vary. From least-squares fitting, the minimum in the reduced $\chi^{2}$ distribution is found for $\gamma \sim 0.25 \pm 0.2$. For the sample of galaxies here, the factor of 2 difference in covering factor implied by the Calzetti ratio is found to be plausible. The CNOC2 galaxies with detected Balmer lines have some preference for an increased covering-factor difference, which perhaps implies that they are undergoing more rapid, 'bursty' star formation than the galaxies Calzetti used in her derivation.

(Babbedge et al. 2005; 443)

This may need a little unpicking for the non-specialist reader. Essentially, my interpretation of the argument in this paper is as follows:

1. Stellar evolutionary history can be inferred from electromagnetic (em) spectral energy distribution (SED);

2. Galactic dust absorbs and reflects some of the em energy; this is called dust extinction;

3. Physicists need to correct for dust extinction if they are to determine stellar histories;

4. Babbedge et al. compared the results of using two photometric codes with another method;

5. They found strong correlation between the results of the two photometric codes;

6. They also found quite a good correlation with the third method, when the poor accuracy of all three methods was taken into consideration;

7. They concluded that the measurement of dust extinction was reliable but not accurate. 
The data for this research was collected via the Canada-France-Hawaii Telescope located on Mauna Kea on Hawaii island - which is an optical/infrared telescope. The outputs from this instrument are presented in the paper by graphs and tables of wavelength intensity distributions et cetera and not as photographs of stars, but, in either case, the output would be meaningless in the absence of the principles of deployment and reading of the instrument and the astrophysical discourse that produces both the experiment and the results presented in the paper and, indeed, the form of the argument presented above. Certainly, the telescope is pointing away from the physicists and at something constituted as the empirical, but just what this is is entirely consumed by an articulation of, on the one hand, physics theory, and, on the other, what Latour and Woolgar (1979) referred to as inscription devices and their principles of deployment and reading. That is to say, the objects of astrophysics - galaxies, the electromagnetic spectrum, galactic dust, and so forth - are constituted and not represented by the practice of astrophysics; real galaxies, electromagnetic radiation, and dust are postulated by a positivist interpretation of the activity and serve to legitimate astrophysics for those of us who make our living in other ways: the myth of reference (Dowling 1998) - forensics. Crucially, the situation in respect of the objects of mathematics as postulated by Duval (2006) - objects that are accessible only via semiotic representations - is identical. Identical, that is, except for the currently unfashionable nature of platonic discourse that would reify them in the way that both everyday and physics discourses reify their objects (note in particular the recent fuss over the Higgs boson ${ }^{1}$ ). Duval's explanation of the particular difficulties experienced by students of mathematics - his forensic push into the empirical world of mathematics education - founders because at least one of the specificities of mathematics that he identifies is revealed as of his own construction. The other specificity - that mathematics, par excellence, deploys a range of semiotic representation systems - is a poor representation of knowledge (to use his own form of argument) as the most casual glance at the physics curriculum will affirm.

\section{Discursive saturation and grammatical modes}

I have constituted astrophysics, as instanced in Babbedge et al. (2005), as deploying two forms of "language," astrophysics theory and inscription devices together with their principles of operation. I want to refer to these two languages as instances of internal and external languages, respectively. Bernstein distinguishes between the two as components of a "language of description" as follows:

Briefly, a language of description is a translation device whereby one language is transformed into another. We can distinguish between internal and external languages of description. The internal language of description refers to the syntax whereby a conceptual language is created. The external language of description refers to the syntax whereby the internal language can describe something other than itself.

(Bernstein 1996; 135-6)

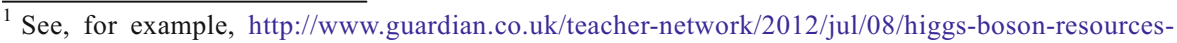
teachers?INTCMP=SRCH.
} 
I am going to use the expression organisational language rather than Bernstein's "language of description." Let us take the organisational language to be constituted by the research language - the translating or representing language - and comprising internal and external languages; this is what I am going to be talking about below. The empirical setting is, referring to the Bernstein extract above, the other language - the language that is to be translated into the research language or, in other words, the language that is to be represented. Referring back to the astrophysics example, then, we might identify the language of astrophysics theory as the internal language and the apparatus of inscription devices and associated principles of operation as the external language. At this point I need to make reference to a construct from my own language, discursive saturation (DS) (see Dowling 1994, 1996, 1998, 2009). I refer to strategies that tend to make the principles of a practice available explicitly within language as high discursive saturation $\left(\mathrm{DS}^{+}\right)$; low discursive saturation $\left(\mathrm{DS}^{-}\right)$refers to the opposite situation in which strategies are tacit. Now the abstract from Babbedge et al. (2005) suggests that both internal and external languages of astrophysics, as constituted in the paper, are characterised by $\mathrm{DS}^{+}$ strategies. I refer to this kind of an organisational language as a metonymic apparatus (Dowling 2009). The astrophysics of Babbedge et al., then, stands in contrast with Bernstein's own language, which tends to remain in the internal. This is certainly explicitly developed, but, as I have argued in Dowling (2009), there is no developed external language - no Bernsteinian telescopes - so that Bernstein's concepts are both too easy and too difficult to operationalise; they do no work in engaging with the empirical. Bernstein's language is a metaphorical apparatus.

SAM is really the opposite kind of thing from Bernstein's language. Its external language - the operationalised/operationalisable content - is explicitly developed; approximately 200 categories are summarised in the glossary presented in Sociology as Method (Dowling 2009) and more have been added since then. I shall present some of this structure - including some new elements - in this paper. The internal language, however, is relatively undeveloped and is summarised in the following sentence: the sociocultural constitutes and is constituted by strategic, autopoietic ${ }^{3}$ action directed at the formation, maintenance and destabilising of alliances and oppositions, the visibility of which, in terms of regularity of practice, is emergent upon the totality of such action and is thereby available for recruitment into subsequent action. Key terms here (though all are important) are: strategy, action, alliance and opposition, and emergence. Essentially, the external language arises and is developed out of the transaction between this internal language and empirical settings. The internal language is a kind of prejudice or bias - a "guiding thread" as Marx (1968 edn) famously described his base/superstructure metaphor - but it is the external language that stands as a legacy of successive engagements. I refer to SAM as a method and in this it perhaps resonates with classic grounded theory (Glaser and Strauss 1965, 1967; Glaser 1992; Martin and Gynnild 2011, see also Dowling 2012);

\footnotetext{
${ }^{2}$ See Dowling (2009) for an explanation of this decision, though the initial paragraphs to the current paper provide some indication of the rationale.

${ }^{3}$ Autopoiesis means self-making.
} 
classic grounded theory is not a theory, but a method and grounded theory is what emerges from its deployment. ${ }^{4}$

At this point, I am in a position to summarise the schema of grammatical modes of organisational languages in diagrammatic form in Table 1 . Three of the categories have already been mentioned. The fourth - fiction - constitutes a strategy that exhibits $\mathrm{DS}^{-}$in respect of both internal and external languages. This mode characterises some approaches in literary criticism - see, for example, Leavis (1950) - but certainly not all: The new historicism of Louis Montrose (1989) and other examples of literary theory would perhaps be better described as deploying metaphorical apparatus.

A crucial point should be made about this schema before I proceed. It will be apparent that it is organised on the basis of discrete, binary-scaled variables and this is generally characteristic of the schemas that comprise the external language of SAM. Essentially, this is a complete space: There are no other possibilities if grammatical modes are conceptualised in this way. This kind of scheme is often criticised as oversimplifying its concepts that, surely, should be constituted as continua. A continuum, however, can be established only where there is a metric and here there is not. ${ }^{5}$ The schemes do not unduly reduce complexity, because any given instance - the astrophysics example, for instance - will generally be revealed as deploying more than one strategy. It will, though, always be possible to identify single strategies by setting the level of analysis low enough. The scheme then serves as a language that can map the practice: where and under what circumstances does it operate as a method, where as a fiction, for example. ${ }^{6}$ The lack of this facility is a key failure of Bernstein's language that, as I have illustrated above, tends to totalise practices: Mathematics, according to Bernstein, is a horizontal knowledge structure with a strong grammar; all of it and in all of its realisations, apparently.

\footnotetext{
${ }^{4}$ The internal language of classical grounded theory is, of course, widely contested (although not by its "mentored" exponents - see Martin and Gynnild 2011), but is it all that important?

${ }^{5}$ The imagination of a continuum where there is no obvious metric is widespread and clearly itself a reduction. It is sometimes introduced with perhaps less culpability in pedagogic texts - see, for example, the reflection on the participant/observer "continuum" in Dowling and Brown (2010) - though it is still doing violence to the concepts.

${ }^{6}$ The accumulation of such codings may enable us to identify a dominant strategy or strategies. Again, however, this is not going to be straightforward because the enumeration of strategies renders all codings to be equivalent. This is a problem to the extent, for example, that the particular prominence or situation of a given instance of, say fiction, may establish it as having far greater semiotic significance than multiple instances of method. None of this is to argue that counting should be avoided at all costs in qualitative research, but it must be handled with care and, even so, will always distort.

${ }^{7}$ Having just argued against the totalising of disciplines alongside the presentation of examples that may be taken to do exactly that, a little further explanation is needed. The examples that I have used are from astrophysics (Babbedge et al.), sociology (Bernstein, Dowling), and literary criticism (Leavis, Montrose). I am referring to these as instances within disciplines rather than as representatives of disciplines. The divergence between Bernstein and Dowling, in sociology, and Leavis and Montose, in literary criticism, makes plain that disciplines are not military institutions, demanding uniformity. Indeed, a closer look at each of these instances would doubtless identify the presence of other strategies, so that even they are not pure instances. This having been said, no extended analysis of these examples is offered and their purpose in this paper is primarily pedagogic. So, I have to admit to a playing on presumed stereotypes that enables me to assume a ready association of physics with inscription devices and internal theory and a common, if unfortunate, association of literary criticism with fiction; unfortunate, perhaps, though I think that fiction (i.e., of my kind) is rather what Leavis was advocating.
} 
Table 1 Grammatical modes of organisational languages (from Dowling 2009)

\begin{tabular}{lll}
\hline & \multicolumn{2}{l}{ Internal Language } \\
\cline { 2 - 3 } External Language & $\mathrm{DS}^{+}$ & $\mathrm{DS}^{-}$ \\
\hline $\mathrm{DS}^{+}$ & metonymic apparatus & method \\
$\mathrm{DS}^{-}$ & metaphoric apparatus & fiction \\
\hline
\end{tabular}

\section{Domains of action}

So how about mathematical organisational language(s)? Michel Foucault described the discipline as "the only discursive practice to have crossed at one and the same time the thresholds of positivity, epistemologization, scientificity, and formalization" $(1972 ; 188)$, but it is not clear just what Foucault may be looking at (or when) in claiming this generalisation. In his own totalising description, above, Bernstein describes mathematics (and logic) as not having empirical referents. This kind of generalisation is rather characteristic of Bernstein's (and sometimes Foucault's) apparent lack of a sense of need of the empirical; the single example of the mathematician John Forbes Nash, whose work in game theory won him a share of the Nobel Prize in Economics in 1994, will suffice to correct him. It is nevertheless the case that a substantial body of mathematical practice can be described as strongly institutionalised $\left(\mathrm{I}^{+}\right)$mathematics that is specialised in respect of both form of expression - deploying the technical terminology and formal notation of mathematics - and content - constituting specifically mathematical objects and processes. Focusing particularly on school mathematics, I described this region of mathematical practice as its esoteric domain (Dowling 1995, 1998, 2009). Because it is the exclusive domain of $\mathrm{I}^{+}$expression and content, it is only within this domain that the principles of mathematical practice can be fully elaborated. Nevertheless, not all of mathematical practice should be described in this way. Considering the level of institutionalisation $\left(\mathrm{I}^{+} / \mathrm{I}^{-}\right)$of form of expression and content separately yields four domains of action as shown in Table 2. In the remainder of this paper I want to illustrate some uses of this scheme and also to develop it.

In Dowling $(1998,2009)$ I described the public domain as the product of a gaze cast by the subject of esoteric domain action beyond $\mathrm{I}^{+}$mathematics, recontextualising non-mathematical practice - such as shopping - by organising it in conformity with mathematical principles. Now, as I have presented the situation here, this formulation is in need of some adjustment, because, insofar as the esoteric

Table 2 Domains of action scheme (from Dowling 2009)

\begin{tabular}{lll}
\hline & Content & \\
\cline { 2 - 3 } Expression & $\mathrm{I}^{+}$ & $\mathrm{I}^{-}$ \\
\hline $\mathrm{I}^{+}$ & esoteric domain & $\begin{array}{l}\text { descriptive domain } \\
\text { public domain }\end{array}$ \\
$\mathrm{I}^{-}$ & expressive domain & P \\
\hline
\end{tabular}


domain comprises essentially self-referential $\mathrm{I}^{+}$expression and content, it should be described as a metaphoric apparatus, which is to say, it has no external language that would enable the gaze. I now want to describe the esoteric domain of school mathematics as an assemblage (see, for example, Turnbull 2000) or perhaps as a rhizome (Deleuze and Guattari 1987) that articulates esoteric domain mathematics as I have described it thus far with pedagogic theory that is more or less explicit $\left(\mathrm{DS}^{+} / \mathrm{DS}^{-}\right)$and that serves to fetch non-mathematical content and expression and organise it in such a way as to align with mathematical content and expression. Here is an example taken from the June 2010 Foundation Tier General Certificate of Secondary Education (GCSE) paper:

A restaurant offers a family discount. The Taylor family have a meal at this restaurant. Before the discount the meal costs $£ 140$. After the discount the cost is $£ 112$. Calculate the percentage discount.

$$
\text { (p. 21, item 22) }{ }^{8}
$$

The nature of the recontextualisation is clear: The parameters are organised so that the cost of the meal before and after the application of the discount is known, whilst the amount of the discount is unknown - rendering this a rather improbable (though not, I suppose, impossible) problem in the restaurant - and the amounts of money involved are arranged so that the answer comes out to a simple figure (20\%). The example is public domain because both the setting - content - and mode of expression are non-mathematical. The next item - from the November 2009 Higher Tier GCSE paper - is coded as descriptive domain because, whilst the setting - content concerns the pricing of newspapers and magazines and is non-mathematical, the text incorporates $\mathrm{I}^{+}$mathematical expression:

The cost of a newspaper is $x$ pence. The cost of a magazine is $£ 1.25$ more than the newspaper. The cost of three of these magazines is the same as the cost of eight of these newspapers.

Show clearly that $3 x+375=8 x$

$$
\text { (p. 4, item 4(a)) }
$$

Here is another item from the June 2010 Foundation Tier paper:

Jack has a box of 100 coloured discs. The discs are Red, Blue, Green and Yellow. The table shows some of the probabilities of choosing a colour.

\begin{tabular}{lllll}
\hline Colour & Red & Blue & Green & Yellow \\
Probability & 0.6 & 0.1 & & 0.1 \\
\hline
\end{tabular}

\footnotetext{
${ }^{8}$ All of the GCSE papers that are referred to in this paper were sourced from the AQA site at http:// web.aqa.org.uk/
} 
Which coloured disc is the most common?

(p. 14, item 15(a))

Essentially, the setting - content - here is $\mathrm{I}^{+}$mathematics - probability - but it is expressed using, primarily, non-mathematical signifiers - coloured discs; this is an expressive domain strategy. ${ }^{9}$ It appears that probability is commonly presented by expressive domain strategies. This is consistent in the 2009 and 2010 GCSE papers and also in the mathematics textbooks that I analysed in some detail some time ago (see Dowling 1995, 1998). Insofar as school mathematics is generally constructed in this way, then access to the esoteric domain in this area is being restricted, possibly explaining why probability is often found to be a difficult topic. Finally, esoteric domain strategies involve both $\mathrm{I}^{+}$expression and content, for example (from the November 2009 Higher Tier paper):

Solve the simultaneous equations $\quad 3 x-2 y=9$

$$
x+4 y=10
$$

(p. 13, item 16)

I am constructing school mathematics, then, as an assemblage of $\mathrm{I}^{+}$expression and content, mathematics per se as a metaphoric apparatus - confined to the esoteric domain - and a pedagogic theory $\left(\mathrm{DS}^{+/}\right)$as a method. It is the latter that enables the mathematical gaze by constituting principles of recognition and realisation of nonmathematical content and expression, so facilitating the construction of the public, descriptive, and expressive domains. Quite clearly school mathematics could be construed differently so as, for example, to incorporate the totality of the school curriculum into a single entity; the establishing of $\mathrm{I}^{+}$and $\mathrm{I}^{-}$strategies in terms of the expression/content distinction is, after all, an analytic decision. It is, however, an analytic distinction that has proved useful in generating productive ways of looking at mathematics and, indeed, at other activities. I have already offered one illustration of this in the description of the pedogising of probability as being largely confined - at least early on (Dowling 1995, 1998) - to the expressive domain and so denying access to the esoteric domain of this topic, a topic that is notoriously intractable for many students. Brantlinger (2011) makes interesting use of this scheme in the interrogation of critical mathematics. In earlier publications I also demonstrated the way in which mathematics for "high-ability" and "low-ability" students was oriented towards the esoteric and public domains, respectively and, further, insofar as the recognition principles of "ability" are a function of socioeconomic class, then school mathematics can be interpreted as a device for translating social class into "ability," providing a mathematical career only for students of high social class/"ability" (see also Dowling 1998, Dowling and Burke 2012).

Leaving mathematics for a moment, the expressive domain can also be constituted as a productive region for autodidactic pedagogic action. Take, for example, the case of learning to play a video game. We may constitute the assemblage of hardware electronics and architecture, game structure and software as the game's esoteric domain. For the most part and for most players there is no direct access to this

\footnotetext{
${ }^{9}$ Of course, the term "probability" appears in the text and this is an $\mathrm{I}^{+}$mathematical term. Strictly speaking, we should say that this item combines expressive domain and esoteric domain strategies, though the incidence of the latter is minimal.
} 
domain. Instead, the player has access to a keyboard or console and a graphic user interface (GUI). There are, of course, many ways to engage with a game, some of which might be described as more destructive than pedagogic. One approach, however, is to attempt to construct the principles of the esoteric domain in the language of the console and GUI, which is to say, in the expressive domain. Indeed, the walkthrough sites that are available for many of these games are formulated in expressive domain text. ${ }^{10}$ To go further, this is precisely what is attempted in a great deal of research activity that I refer to as forensics. Essentially, the social researcher is generally constructing an expressive domain language in an attempt to represent the esoteric domain of their object setting. In the more general language of social research, the researcher is deploying or constructing an etic language contrasting with the emic language of the setting itself. Under such circumstances, the expressive domain, etic language constitutes the external language of their research, whilst the general principles or approach and so forth constitute their own esoteric domain and internal language. A switch will have been noticed here. The etic language is indeed constituted in an expressive domain from the perspective of the activity of the empirical setting in and of itself - but is now the descriptive domain of the activity of the social researcher and the empirical and research activities each constitute the public domain of the other. The two activities - the setting activity and the social research activity - confront each other as is illustrated in Fig. 1.

The configuration of the two activities in Fig. 1 presents the etic language of the setting's expressive domain and the research descriptive domain as a representation of the setting activity itself, so that the research public domain is constituted as the same thing as the setting esoteric domain. The research, in this configuration, is attempting to discover how the setting really works as if a reality unmediated by semiosis was available to the researcher. ${ }^{11}$ This is an illustration of what I have referred to above as forensics. By contrast, my constructive description approach incorporates the objects of a language entirely within itself so that the configuration is more appropriately presented as in Fig. 2. ${ }^{12}$ Taking anthropology as presented by Clifford Geertz (1988, 1977 (2000 edn)) as an example, the research may start out as the kind of autodidactics that I described in respect of the video game, which is to say, the anthropologists' attempts to apprentice themselves into the setting; traditionally, this is likely to involve, amongst other things, learning the local language. The initial phase, then might be described as the anthropologist attempting to establish an expressive domain for the setting by "being there" in the field. Ultimately, however, the anthropologist must present their work to the academy, as Geertz puts it:

In itself, Being There is a postcard experience ('I've been to Katmandu - have you?'). It is Being Here, a scholar among scholars, that gets your anthropology read ... published, reviews, cited, taught.

(Geertz 1988; 130; ellipsis in original)

\footnotetext{
${ }^{10}$ See, for example, the walkthroughs on Stella's Tomb Raider Site at http://tombraiders.net/.

${ }^{11}$ Or, alternatively, as if alternative semiotic mediations were somehow comparable or mutually compensatable so that their respective specificities might be taken out of the equation in a kind of triangulation process.

12 These and other configurations of the domains of action scheme arose in discussion with Soh-young Chung (2011), Russell Dudley-Smith and Jeremy Burke. Dudley-Smith and Burke are now completing their doctoral theses at the Institute of Education, University of London.
} 


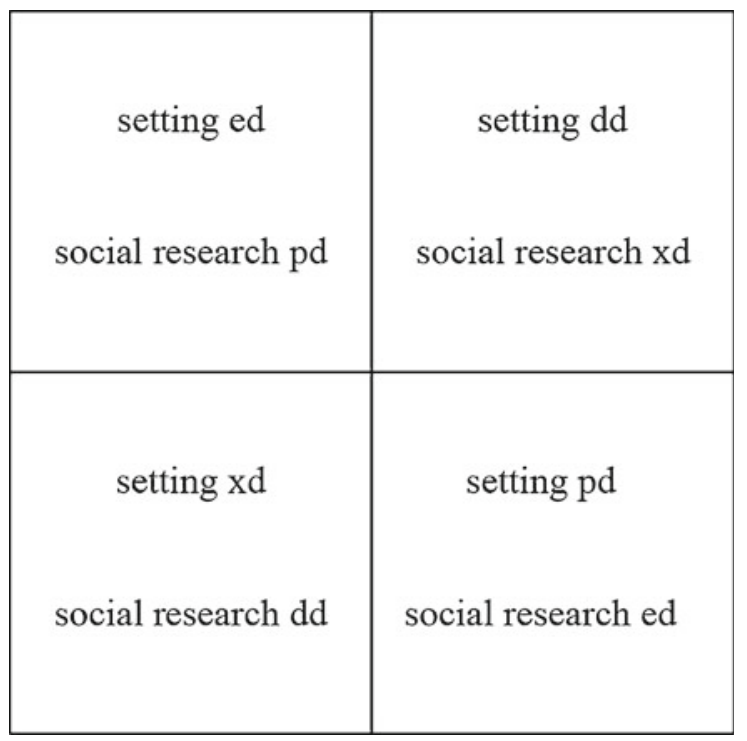

ed = esoteric domain; $\mathrm{dd}=$ descriptive domain $; \mathrm{xd}=$ expressive domain; $\mathrm{pd}=$ public domain Fig. 1 Domains of action - two activities in forensic relation

It is the social and cultural capital of anthropology that constitutes its esoteric domain and that constitutes its interpretations as substantive recontextualisations, and a similar argument explains why the school mathematics classroom is not the place to learn how to do your shopping.

\section{Modes of recontextualisation}

I have, thus far in this paper, said very little about the nature of recontextualisation other than to claim that it involves a transformation of some kind. I want to make another short digression to introduce another SAM scheme that lays out modes of recontextualisation in terms of discursive saturation. This is shown in Table 3.

Pierre Bourdieu (1977) provides an excellent illustration - again from anthropology - of what can happen when $\mathrm{DS}^{+}$strategies (I am using my language, not Bourdieu's) are deployed on $\mathrm{DS}^{-}$practice. Bourdieu critiques the analysis of gift exchange in a non-industrial society by structuralist anthropology. ${ }^{13}$ As Bourdieu argues, the anthropologist may write the society's rules, but the real power plays are always going to go on in the interstices. The presentation of a gift, for example, may require that another gift be made in exchange, but this leaves open the precise nature of the gift (it cannot be the same gift simply given back) and the

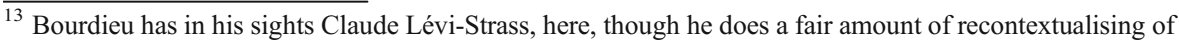
the latter's work. 


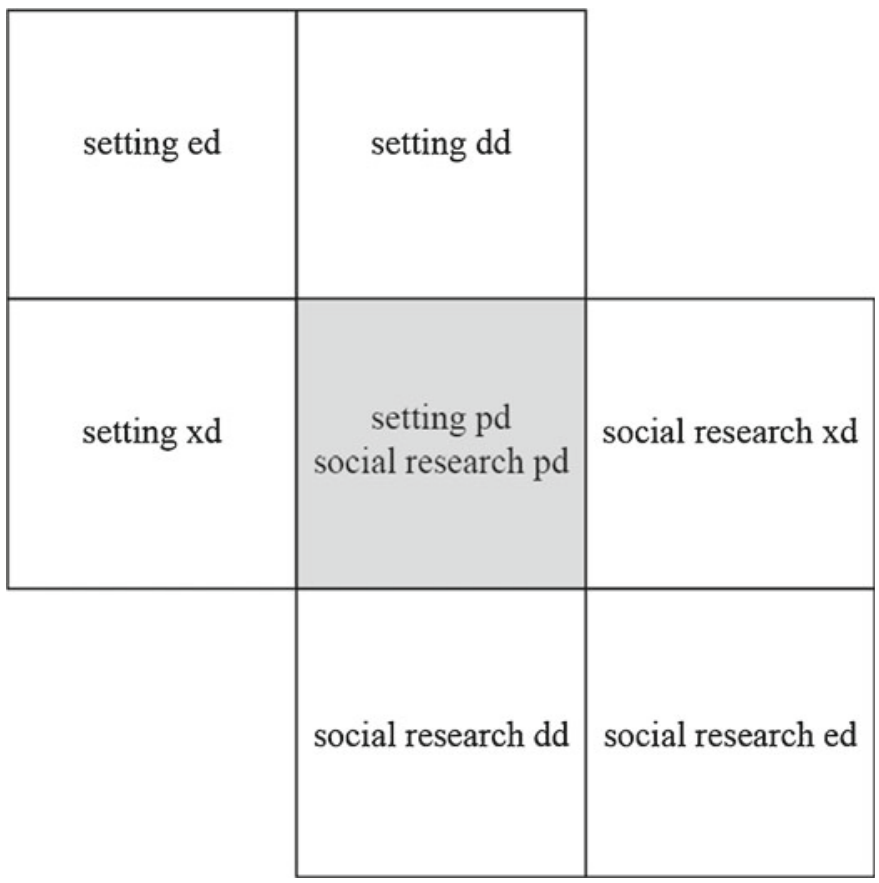

ed = esoteric domain; $\mathrm{dd}=$ descriptive domain; $\mathrm{xd}=$ expressive domain; $\mathrm{pd}=$ public domain Fig. 2 Domains of action - two activities in relation of constructive description

period of time that may elapse before the exchange is made. Indeed, the extending of the period may raise a doubt as to whether the exchange gift will ever be forthcoming. In respect of the anthropology of myth, he warns:

The science of myth is entitled to describe the syntax of myth, but only so long as it is not forgotten that, when it ceases to be seen as a convenient translation, this language destroys the truth that it makes accessible. One can say that gymnastics is geometry so long as this is not taken to mean that the gymnast is a geometer.

(Bourdieu 1990; 93)

Indeed no: Euclid on the beam sounds excruciating!

Table 3 Modes of recontextualisation

\begin{tabular}{lll}
\hline & \multicolumn{2}{l}{ Recontextualised Practice } \\
\cline { 2 - 3 } Recontextualising Strategy & $\mathrm{DS}^{-}$ & $\mathrm{DS}^{+}$ \\
\hline $\mathrm{DS}^{-}$ & $\begin{array}{ll}\text { improvising } \\
\text { rationalising }\end{array}$ & $\begin{array}{l}\text { de-principling } \\
\text { re-principling }\end{array}$ \\
\hline
\end{tabular}


The "science of myth" would seem to involve the deployment of rationalising recontextualisation and it seems that school mathematics often recontextualises domestic and other activities in this way (see the examples in Dowling 1998, 2009). The recontextualising of the restaurant bill introduced earlier also involves rationalising because the numerical elements of the setting are first fetishized and then moved around so as to insert the unknown in a place that is determined by the requirements of the assessment. What I am doing in this paper is deploying $\mathrm{DS}^{+}$strategies in the recontextualising of activities - mathematics, anthropology that themselves involve substantial $\mathrm{DS}^{+}$strategies and, to this extent, I am reprincipling. My recontextualisation of the playing of video games, however, is more like rationalising. Insofar as the setting in Figs. 1 and 2 is predominantly characterised by $\mathrm{DS}^{-}$strategies, then the regard of the social scientist by the setting can be described as de-principling. Indeed, this is also the impact of the recontextualising of educational research in official documents, which generally quote selectively, so losing the principled discourse that will have generated the extracts. Unfortunately, academic authors sometimes have a tendency to do the same violence to other research that they cite. Finally, the category, improvising, characterises what Lévi-Strauss (1972) referred to as "bricolage" - recruiting what is at hand in a semiotic or material construction or production - also, perhaps, some aspects of improvisation in jazz. To what extent might we construe $\mathrm{DS}^{-}$ pedagogic theory as constituting an improvising gaze in the recognition principles of its fetch strategies, the mathematical realisation principles effecting rationalising or reprincipling recontextualisation?

In one way or another, when one activity casts its gaze on another, the outcome will always involve recontextualisation. This, of course, raises serious difficulties for any notion of functional or transferrable skills, yet the functionality of mathematics education is generally high on the list of priorities for the school curriculum. The Australian Curriculum Assessment and Reporting Authority (ACARA), for example, stresses that the mathematics curriculum should

... educate students to be active, thinking citizens, interpreting the world mathematically, and using mathematics to help form their predictions and decisions about personal and financial priorities.

(ACARA quoted by Sullivan 2011)

The imposition of mathematical principles on decision making is always rationalising or (one suspects less frequently) re-principling recontextualisation: The world is frequently rationalised mathematically or by other $\mathrm{DS}^{+}$practices, but, in practice, decisions seem often to be apparently successfully formulated on the basis of what is better described as improvisation as, for example, many of the approaches reported in Lave et al. (1984) illustrate. Even the natural sciences are not always as rational as we might think (see, famously, Latour and Woolgar 1979, and also Knorr Cetina 1999, Collins and Pinch 1998). What I have elsewhere (Dowling 2007, 2009) referred to as mathematicoscience is nevertheless hegemonic in educational policy discourse. Here is the opening statement of the UK National Curriculum for Secondary Mathematics: 
Mathematical thinking is important for all members of a modern society as a habit of mind for its use in the workplace, business and finance, and for personal decision-making. Mathematics is fundamental to national prosperity in providing tools for understanding science, engineering, technology and economics. It is essential in public decision-making and for participation in the knowledge economy. Mathematics equips pupils with uniquely powerful ways to describe, analyse and change the world. ${ }^{14}$

In this statement, mathematics is presented as being entirely for something other than itself and this is presented in the form of the myth of participation (Dowling 1998), which is to say, mathematics is a necessary facility for participation in the nonmathematical activities cited. Now, my analysis of recontextualisation and the examples given here and elsewhere (in particular in Dowling 1998, 2007, and Dowling and Burke 2012) are sufficient to call into serious question the viability of the this proposition and, indeed, to reveal the mythical nature of the myth of participation. Suspending this scepticism, however, it is instructive to deploy the domains of practice scheme in an analysis of GCSE mathematics papers in order to assess the extent to which they do indeed prioritise practical mathematics - mathematics for something other than itself. I used the Foundation- and Higher-Tier papers from November and June 2009 and June 2010 that were available at the time on the AQA site ${ }^{15}$ and analysed the items in terms of the four domains of action. As I have indicated above, the setting for esoteric and expressive domains is $\mathrm{I}^{+}$mathematical content, differing in respect of the mode of expression, and the setting for the public and descriptive domains is $\mathrm{I}^{-}$content (nonmathematical), again differing in that the descriptive domain incorporates $\mathrm{I}^{+}$expression, whilst the public domain does not. In coding, I used the item - differentiated by question number - as the unit of analysis. ${ }^{16}$ There were two papers for each tier for each sitting, giving six Foundation papers and six Higher papers; the results in Table 4 are aggregated for the two tiers, there being no substantial differences between sittings.

As I have indicated, the esoteric and expressive domains both have esoteric domain settings and so signify the esoteric domain and, similarly, the descriptive and public domains signify the public domain. Focusing, then, on the esoteric/expressive and descriptive/public totals (in bold in the table) there are approximately twice as many esoteric domain settings in these papers as there are public domain settings - a little less on the Foundation Tier, a little more on the Higher Tier. When combined with the more or less transformative impact of recontextualisation on public domain settings - illustrated here only via the restaurant item, but consistent throughout these items - it seems clear that there is a substantial mismatch between the summative assessment of the

\footnotetext{
$\overline{14}$ http://www.education.gov.uk/schools/teachingandlearning/curriculum/secondary/b00199003/mathematics/ks3; text updated 25th November 2012; retrieved 11th July 2012. The same text prefaces both Key Stages 3 and 4, although the emphasis on the non-mathematical is, interestingly, comparatively absent from the primary phase programme of work.

${ }^{15}$ AQA (http://web.aqa.org.uk/) describes itself as "an educational charity" that is "the leading provider of GCSEs and A-levels" and claims that their "qualifications are used in most secondary schools and colleges in England, Wales and Northern Ireland ..." (http://www.aqa.org.uk/about-us).

${ }^{16}$ Most of the items were organised in parts $(a, b, c, \ldots)$, but I found that the parts of any given question could validly be coded in the same way.
} 
Table 4 GCSE Papers by tier and domain of action

\section{Domain of Action}

\begin{tabular}{lrrrrrrr} 
Tier & ed & $\mathrm{xd}$ & $\mathrm{dd}$ & $\mathrm{pd}$ & $\mathbf{e d}+\mathbf{x d}$ & $\mathbf{d d}+\mathbf{p d}$ & Total \\
\hline Foundation & 80 & 14 & 31 & 21 & $\mathbf{9 4}$ & $\mathbf{5 2}$ & 146 \\
Higher & 98 & 8 & 30 & 13 & $\mathbf{1 0 6}$ & $\mathbf{4 3}$ & 149
\end{tabular}

Aggregate numbers of items on AQA Papers 1 and 2 for each of June \& November 2009 and June 2010; $\mathrm{ed}=$ esoteric domain; $\mathrm{xd}=$ expressive domain; $\mathrm{dd}=$ descriptive domain; $\mathrm{pd}=$ public domain

National Curriculum as represented in these papers and the stated aims of the curriculum: As central as practical mathematics may be in the stated aims of the curriculum, it is clearly rather sidelined in these assessment instruments.

\section{Mathematics and the esoteric domain}

Practical mathematics is not all there is to the school curriculum. Sullivan (2011), amongst many others, asserts that both "practical" and "specialised" mathematics should be present, though with the former dominating. In my terms, specialised mathematical knowledge ${ }^{17}$ is constituted in the esoteric domain and finally in this paper I want to put some detail onto this domain. Duval (2006), as has already been mentioned, constitutes mathematics in terms of a "range of semiotic representation systems" ( $p$. 108 ) and the representation to which he is referring is of mathematical objects. This keeps us within the esoteric domain and is perhaps consistent with Bernstein's characterisation of mathematics as a "horizontal knowledge structure." It is clearly the case that we can topicalise mathematics (algebra, trigonometry, probability, set theory, and so forth) and we can use what Gunther Kress (2003) and others refer to as different "modes of representation" in mathematical text; I have, of course, already rejected the term "representation" for what I can now refer to as esoteric domain or public domain objects. I do not find it helpful to describe mathematics in either of these ways. Rather, I want to describe the mathematical esoteric domain of school mathematics as itself consisting of an assemblage of strategies that includes: i) discursive definitions, principles, theorems, and so forth; ii) visual exemplars, most obviously in the area of geometry; iii) formal nomenclatures (the decimal representation of number, for example) and heuristics; and iv) instrumentation (calculators, computers, geometric instruments, and so forth). Now this empirically based list can be reconceptualised as a complex apparatus that exhibits variation in semiotic mode - discursive (available within language)/non-discursive (not available within language) - and action - interpretive/procedural. This gives rise to the schema in Table 5.

\footnotetext{
${ }^{17}$ I announced at the head of this paper that I would take "knowledge" as my empirical setting. This is something of a pragmatic (or perhaps pedagogic) recontextualisation of my own position as I generally reject the fetishizing of knowledge (or of any "body" of knowledge) as an object that is available for analysis, though texts associated with that which is often fetishized - mathematics textbooks with mathematical knowledge, for example - are clearly available as empirical objects. I have addressed this issue in Dowling 1998 and 2009 (c. 8) and in my critique of Bernstein in Dowling (2009 c. 4), so I will not pursue it here, but will retain the pragmatic recontextualisation as serving my present purpose.
} 
Table 5 Modality of esoteric domain strategy

\begin{tabular}{|c|c|c|}
\hline \multirow[b]{2}{*}{ Mode of Action } & \multicolumn{2}{|c|}{ Semiotic Mode } \\
\hline & Discursive & Non-discursive \\
\hline Interpretive & theorem & template \\
\hline Procedural & procedure & operational matrix \\
\hline
\end{tabular}

Now, quite clearly, the categories constituted in Table 5 refer to general/ generalisable aspects of the esoteric domain; a template would be of little use if it constituted a unique instance. These general modes are then repeated as local instances, giving rise to a three-dimensional schema, represented in Table 6. I'll take a GCSE item, shown in Fig. 3, to illustrate the scheme. The statements at the head of the item and the reference to the "angle, marked $x$, on the diagram" are instances of localised theorems, which is to say, enunciations. The diagram itself is a graph - a local, non-discursive, interpretive strategy. The rest of the text consists of instructions that pertain to assessment and so we might say that this relates to "pedagogic theory." The statement "Not drawn accurately" is clearly important in disabling the deployment of an operational matrix - a protractor - in solving the item via an operation (measuring the angle on the diagram).

Denied the operational mode, we need to consider what the esoteric domain has to offer that might be of assistance to us. Essentially, we are looking for general interpretative or procedural strategies. One approach is to look for templates that resonate with the diagram in the task and we might refer to this as a procedure or, with direct reference to the problem graph, as a protocol. Three resonant templates are shown in Figs. 4, 5, and 6. The titles of each figure include reference to a theorem. The advantage of the template format, however, is that we can potentially recognise the template in the problem graph. I have made this easier for those who are at some distance from their study of school geometry by constructing the templates by selecting from my digital drawing (in Adobe Illustrator) of the problem graph; this strategy is, of course, the opposite way around from the approach that a GCSE candidate would take in solving the problem. It should be quite easy to visualise the three templates in the graph, so we can conclude that: angle $A C D=40^{\circ}$ (from Fig. 4); angle $D A E=40^{\circ}$ (from Fig. 5); so angle $C A E=32^{\circ}+40^{\circ}=72^{\circ}$; and, finally, angle $x=180^{\circ}-(40+72)^{\circ}=68^{\circ}$ (from Fig. 6). These statements are all enunciations or localised theorems.

Table 6 Modality of general and local esoteric domain apparatus

\begin{tabular}{lll}
\hline & \multicolumn{2}{c}{ Semiotic Mode } \\
\cline { 2 - 3 } Mode of Action & Discursive & Non-discursive \\
\hline General & & \\
Interpretive & theorem & template \\
Procedural & procedure & operational matrix \\
Local & & \\
Interpretive & enunciation & graph \\
Procedural & protocol & operation \\
\hline
\end{tabular}


$A B C D$ is a cyclic quadrilateral.

$A E$ is a tangent at $A$.

$C D E$ is a straight line.

Angle $C A D=32^{\circ}$

Angle $A B D=40^{\circ}$

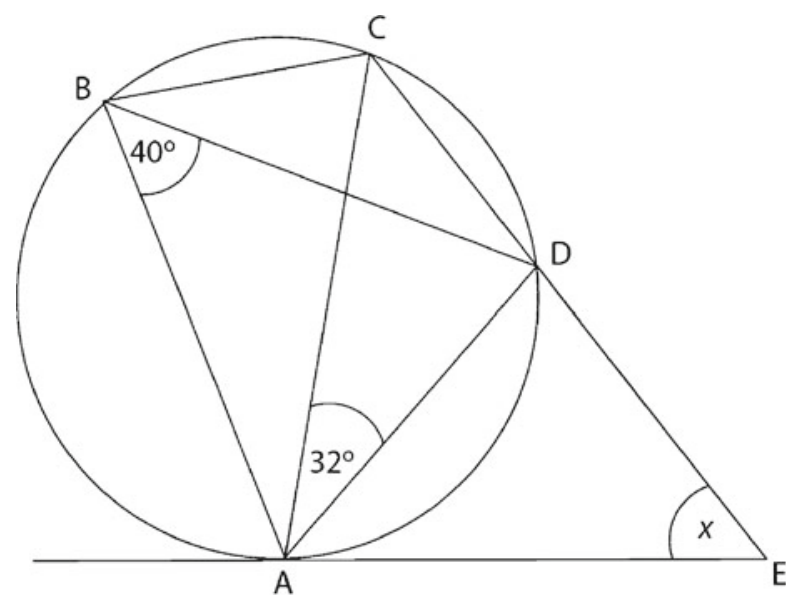

Work out the size of angle $A E D$, marked $x$, on the diagram.

You must show your working.

Give reasons for any angles you work out.

Fig. 3 GCSE Paper 1H June 2010 redrawn from item 24

There are, of course, alternative esoteric domain strategies that might be deployed in solving this problem. These include reliance on theorems without visualising the templates. Also, alternative templates might be selected, including, for example, the one in Fig. 7, which might be deployed in proving or sidestepping the theorems corresponding to Figs. 2 and 5 or validating the templates, should these

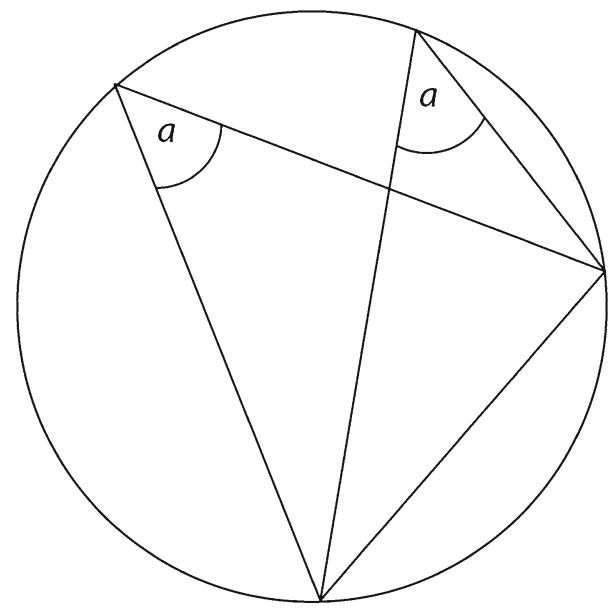

Fig. 4 Geometrical template: same segment theorem 


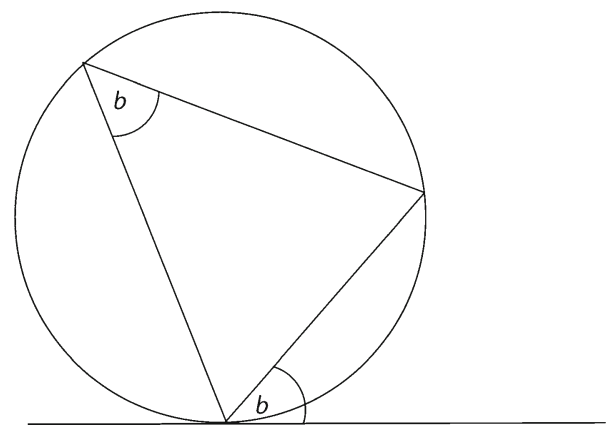

Fig. 5 Geometrical template: alternate segment theorem

theorems/templates not come clearly to mind. All of these templates articulate more theorems than are likely to be required in any given situation. Figure 7, for example, connotes (for me; doubtless, I've missed some) the following theorems:

1. A circle is the set of points that are equidistant from a fixed point that is its centre;

2. There are two tangents to a circle from any point outside the circle;

3. These tangents are of equal length;

4. The tangents are perpendicular to the radius of the circle at the point at which they touch the circle;

5. The two tangents to a circle from a point outside of the circle, the line joining this point with the centre of the circle, and the radii of the circle at the points at which the tangents touch the circle form two congruent, right-angled triangles;

and also the following operational matrix:

6. Circles and line segments may be constructed by straight edge and compasses (or using draw software on a computer).

So what is the point of this expansion of language relating to the esoteric domain of school mathematics; what is its use-value? Earlier, I cited Duval's (2006) construction of

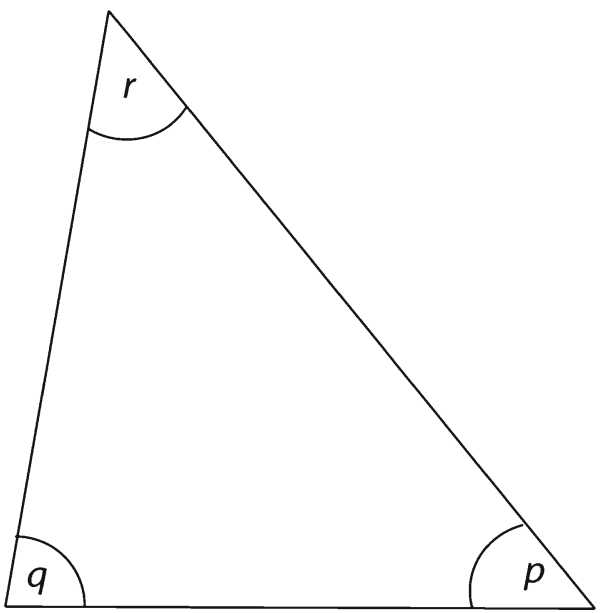

Fig. 6 Geometrical template: internal angles of a triangle sum to $180^{\circ}$ 


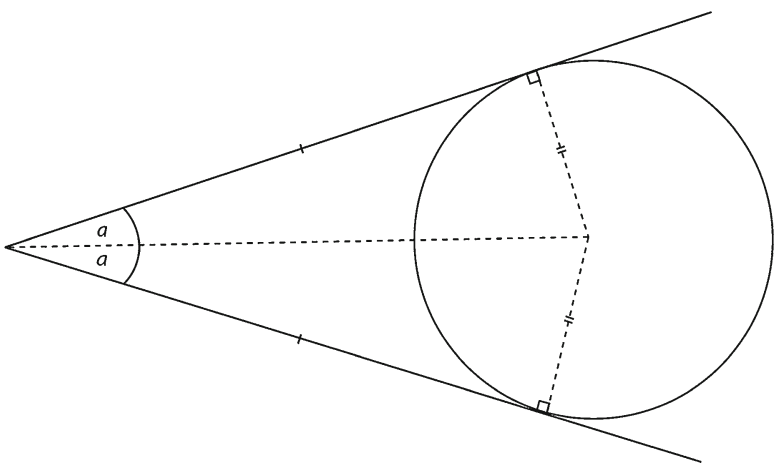

Fig. 7 Geometrical template: two tangents from a point to a circle

mathematical knowledge as comprising mathematical objects that are represented or mediated by a range of different semiotic systems. The object-oriented approach requires the learner to acquire the objects through engagement with their representations in the various systems and this acquisition might be referred to as understanding; Duval identifies some of the difficulties that this entails for the learner. SAM's approach, by contrast, denies the value of positing objects that are outside of semiosis. Rather than attempt the transmission of such mythical entities, SAM emphasises the assemblage of esoteric domain strategies that are available for recruitment in mathematical activity. This bears some similarity with Polya's (1946) listing of heuristics, which, in my scheme, are classified as procedures. It would, of course, be possible to articulate these procedures and, indeed, the theorems, templates, and operational matrices into some kind of more or less coherent discourse, although this would involve the rationalising of templates and operational matrices. Even if this were to be achieved, however, the acquisition of the discourse would have to be achieved in a single move, rather like Foucault's (1972) all-in-one-go vision of formalised mathematics. Since this is not possible (in the high school, anyway), understanding - the meanings that can be provided only within the system as a whole must be perpetually deferred. We know, in fact, that this is the case: How is it that elementary school students can possibly be said to "understand" the natural numbers without a grasp of the Peano axioms (probably unfamiliar even to their teachers)? What the scheme in Table 6 offers is the basis for a curriculum of strategic, mathematical resources. In the presentation of such a curriculum, understandings will come and will go and will change with the partial totalities that are acquired along the way and will always of necessity be localised to the individual at a particular point in their mathematical education. Many of these understandings will be $\mathrm{DS}^{-}{ }^{-18}$ This may be a shock to those who, like Foucault, see mathematics as necessarily already fully formalised, but perhaps this is a necessary shock. SAM's approach also, incidentally, strikes at the heart of Bernstein's totalising.

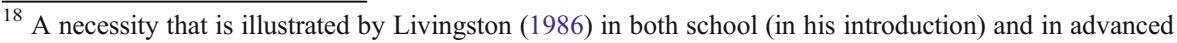
mathematics.
} 


\section{Conclusion}

In this paper I have, first, offered an alternative to what I am referring to as forensics. The latter presents its objects as lying, somehow, beyond its own artifice, beyond semiosis. In astrophysics, the introduction of forensics - we are finding more and more about the universe, which is real and which was around before us and will be around after we're gone - may settle those who live in the world of quotidian commonsense and perhaps ease the way for funding applications for very big science, but it won't necessarily have much of an impact on the day-to-day working of jobbing astrophysicists. In school mathematics, by contrast, forensics seduces, but then confuses and ultimately betrays with the promise of mythical objects to be acquired but, like the carrot dangling from the donkey's stick or like the greyhound's hare, must always be just out of reach - Tantalus in the classroom. Constructionism, in one form or another, is not new, of course; indeed it's exactly as old as its positivist other, the two dancing their perpetual tango, penetrating each other's spaces, but never touching except, tellingly, by holding hands. The dance is, of course, infertile. So I will rely on pragmatic principles to determine the value of the approach that I present - the organisational language, SAM.

I have presented a number of SAM constructs in this paper. The grammatical modes scheme distinguishes between strategies that exhibit high and low discursive saturation in respect of the internal and external languages of an activity. This scheme, together with the domains of action scheme, enables me to describe school mathematics as an assemblage comprising a self-referential, mathematical, esoteric domain as a metaphoric apparatus, and pedagogic theory that may vary in terms of $\mathrm{DS}^{+/}$and that facilitates the gaze that constitutes the other domains of action. This scheme together with the modes of recontextualisation scheme organises the fundamental action of recontextualisation that is the inevitable outcome when one activity regards another, the positivity of an activity being the visible regularity of social relations and cultural practices - in terms of alliances and oppositions - that is emergent upon the totality of strategic, autopoietic action that constitutes the sociocultural and that SAM endeavours to describe. Quite apart from its other virtues, constructing the world in this way reveals and describes the mendacious nature of any attempt to totalise or essentialise activity or knowledge such as mathematics.

I have attempted to illustrate the pragmatic value of SAM by illustrating the way in which we might productively think about autodidactic pedagogy and about the action of research activity (for example, in anthropology) as initially autodidactic and subsequently constituting its own gaze. This formulation may be of value in thinking about and constructing curricula in research activities. The modes of recontextualisation scheme provides some indication of the specific impact of the recontextualisating action of $\mathrm{DS}^{+}$and $\mathrm{DS}^{-}$strategies deployed on $\mathrm{DS}^{+}$and $\mathrm{DS}^{-}$ practice and this, in particular, describes the rationalising action of formalisation on non-discursive mathematical templates and operational matrices. The deployment of the domains of action scheme reveals as ideological the UK National Curriculum claim that mathematics is primarily about something other than itself by revealing (through an analysis of GCSE papers) both that summative assessment at $16+$ is overwhelmingly concerned with esoteric domain settings and that even where public 
domain settings are introduced, the effect of recontextualisation brings these settings into mathematics rather than, for example, taking mathematics out into the world. The latter effect would require a strategy operating in the opposite direction: not a push from mathematics, but a fetch from the supermarket. I have also given further consideration to the nature of the esoteric domain as an assemblage of strategic resources (in Table 6), arguing that organising the curriculum on the basis of this assemblage might overcome some of the problems associated with the forensic approach.

I earlier explained my use of the word "for" in my title. The term left unexplained is "fractal." It is there because the method that I am presenting is characterised as independent of the level of analysis at which it is deployed. I have hinted at this in my initial explanation of the use of the SAM schemes: You need to reduce the level of analysis sufficiently in order to identify a unique mode, shall we say, metaphoric apparatus, metonymic apparatus, method, or fiction. Moving back up the levels of analysis will reveal any practice as hybrid, but the same language can be used consistently. Indeed, SAM is also capable of describing its own actions as I have also illustrated. For example, it operates, essentially, as a method. Its internal language - or prejudice - is constituted as a single sentence concerning strategy, action, alliance and opposition, and emergence - its external language - or dynamic legacy comprises the SAM schemes, some of which are presented (and one of which is developed) in this paper. Primarily, SAM's analytic action is $\mathrm{DS}^{+}$, so its mode of recontextualising tends to be rationalising or re-principling. The diagrams in Tables 1 and 2, Figs. 1 and 2, Table 3, Tables 5 and 6 are graphic renderings of what can quite easily and unambiguously be rendered discursively; they are theorems rather than templates. Nevertheless, there must remain substantial $\mathrm{DS}^{-}$content. I cannot, by definition, quite get at any of it, but I can point to, for example, the necessary uncertainty that is involved in the coding generating Table 4 and the semiotic haze that must obscure all definitions of terms.

So here is a fractal language for mathematics - SAM. Most crucially, however, its rationalising, re-principling, de-principling, and improvising actions of recontextualisation must disable the social relations that characterise - albeit on an emergent basis - the alliances that constitute its object as their own. Once discourse reaches its limits, other modes must take over.

Acknowledgments I am grateful to Soh-young Chung and Russell Dudley-Smith for their critical interrogation of the first draft of this paper and to the organisers of and participants in the Madif7 conference, Stockholm University, January 2010, where some of the ideas in the paper were first opened up in my plenary address.

Open Access This article is distributed under the terms of the Creative Commons Attribution License which permits any use, distribution, and reproduction in any medium, provided the original author(s) and the source are credited.

\section{References}

Babbedge, T. S. R., Whitaker, R., \& Morris, S. (2005). 'Can a photometric redshift code reliably determine dust extinction? Monthly Notices of the Royal Astronomical Society, 361(2), 437-450.

Bernstein, B. B. (1996). Pedagogy, symbolic control and identity. London: Taylor \& Francis. 
Bernstein, B. B. (1999). Vertical and horizontal discourse: an essay. British Journal of Sociology of Education, 20(2), 158-173.

Bourdieu, P. (1977). Outline of a theory of practice. Cambridge: CUP.

Bourdieu, P. (1990). The logic of practice. Cambridge: Polity.

Brantlinger, A. (2011). Rethinking critical mathematics: a comparative analysis of critical, reform, and traditional geometry instructional texts. Educational Studies in Mathematics, 78(3), 395-411.

Carr, W., \& Kemmis, S. (1986). Becoming critical: Education, knowledge and action research. Oxford: RoutledgeFalmer.

Chung, S-y. (2011). 'The modality of the textual institutionalisation of literary studies: towards a sociology.' Sociological Research Online. 16/3/3. 1-14.

Collins, H., \& Pinch, T. (1998). The Golem: What you should know about science. London: CUP.

Deleuze, G., \& Guattari, F. (1987). A Thousand Plateaus: Capitalism and schizophrenia. Minneapolis: University of Minnesota Press.

Dowling, P. C. (1994). Discursive saturation and school mathematics texts: A strand from a language of description. In P. Ernest (Ed.), Mathematics, education and philosophy: An international perspective. London: Falmer Press.

Dowling, P. C. (1995). 'A Language for the Sociological Description of Pedagogic Texts with Particular Reference to the Secondary School Mathematics Scheme SMP 11-16'. Collected Original Resources in Education. 19(2). No journal page numbers.

Dowling, P. C. (1996). A sociological analysis of school mathematics texts. Educational Studies in Mathematics, 31(4), 389-415.

Dowling, P. C. (1998). The sociology of mathematics education: Mathematical myths/pedagogic texts. London: Falmer Press.

Dowling, P. C. (2007). Quixote's science: Public heresy/private apostasy. In B. Atweh, A. C. Barton, M. C. Borba, N. Gough, C. Keitel, C. Vistro-Yu, \& R. Vithal (Eds.), Internationalisation and globalisation in mathematics and science education (pp. 173-198). Dordrecht: Springer.

Dowling, P. C. (2009). Sociology as method: Departures from the forensics of culture, text and knowledge. Rotterdam: Sense Publishers.

Dowling, P. C. (2012). 'Being Barney Glaser.' Grounded Theory Review. 11(2). no page numbers.

Dowling, P. C., \& Brown, A. J. (2010). Doing Research/Reading Research: Re-interrogating education. London: Routledge.

Dowling, P. C., \& Burke, J. (2012). Shall we do politics or learn some maths today? Representing and interrogating social inequality. In H. Forgasz \& F. Rivera (Eds.), towards equity in mathematics education: Gender, culture, and diversity (pp. 87-104). Heidelberg: Springer.

Duval, R. (2006). 'A Cognitive Analysis of Problems of Comprehension in a Learning of Mathematics.' Educational Studies in Mathematics, 61(1/2), 103-131.

Foucault, M. (1972). The archaeology of knowledge. London: Tavistock.

Geertz, C. (1977). The interpretation of cultures (2000 ed.). New York: Basic Books.

Geertz, C. (1988). Works and lives: The anthropologist as author. Cambridge: Polity.

Glaser, B. (1992). Basics of grounded theory analysis: Emergence versus forcing. Mill Valley: Sociology Press.

Glaser, B., \& Strauss, A. (1965). Awareness of dying. Chicago: Aldine Publishing.

Glaser, B., \& Strauss, A. (1967). The discovery of grounded theory: Strategies for qualitative research. New York: Aldine Publishing Company.

Knorr Cetina, K. (1999). Epistemic Cultures: How the sciences make knowledge. Cambridge: Harvard University Press.

Kress, G. (2003). Literacy in the new media Age. London: Routlege.

Latour, B., \& Woolgar, S. (1979). Laboratory life: The social construction of scientific facts. Beverly Hills: Sage.

Lave, J., Murtaugh, M., \& de la Rocha, O. (1984). The dialectic of arithmetic in grocery shopping. In B. Rogoff \& J. Lave (Eds.), Everyday cognition: its development in social context. Cambridge: Harvard University Press

Leavis, F. R. (1950). The great tradition. New York: George W. Stewart, Publisher Inc.

Lévi-Strauss, C. (1972). The Savage Mind (La Pensée Sauvage). London: Weidenfeld \& Nicholson.

Livingston, E. (1986). The ethnomethodological foundations of mathematics. London: RKP.

Martin, V. B. \& Gynnild, A. (Eds). (2011). Grounded Theory: The philosophy, method and work of Barney Glaser. Boca Raton. BrownWalker Press.

Marx, K. (1968). Preface to a contribution to the critique of political economy. In K. Marx \& F. Engels (Eds.), Selected works. London: Lawrence \& Wishart. 
Montrose, L. A. (1989). Professing the Renaissance: The poetics and politics of culture. In H. A. Veeser (Ed.), The new historicism (pp. 15-36). New York: Routledge.

Polya, G. (1946). How to Solve it: A new aspect of mathematical method. Princeton: Princeton University Press.

Rorty, R. (1989). Contingency, irony and solidarity. Cambridge: Cambridge University Press.

Schön, D. A. (1987). Educating the reflective practitioner: Toward a new design for teaching and learning in the professions. San Francisco: Jossey-Bass.

Sullivan, P. (2011). Teaching mathematics: Using research-informed strategies. Camberwell: Australian Council for Educational Research.

Turnbull, D. (2000). Masons, tricksters and cartographers: Comparative studies in the sociology of scientific and indigenous knowledge. London: Routledge. 